\title{
Building Damage Level According to Distance Image Contrast.
}

\author{
Liwei Chen \\ College of Computer Science and Technology, SouthWest University of Science and Technology, \\ Mianyang,621010,China
}

\begin{abstract}
Estimation method of building damage level was introduced for the accurate and effective estimation of damage extent and relief goods demand according to long-distance image contrast. In order to obtain completion degree of building edge extracted from longdistance images before and after disaster, the concentration ratio was analyzed with Hough transformation. Based on the maximum posterior probability, estimation method of affected population was designed to accurately estimate victim population, which can be directly reflected by fugitive population. Moreover, on basis of escape route and fugitive population, demand assignment algorithm by backward calculation was designed to improve rescue efficiency.
\end{abstract}

Key Words: emergency rescue, demand estimation, Hough transformation, maximum posterior probability

\section{Introduction}

Relief goods are in objective demand after disaster, while the understanding of demand condition is deficient. After disaster, information channel is obstructed and interrupted; affected people, with fear, are hurried to escape from the disaster. Therefore, it is difficult to know precise damage extent and its distribution in short time. This deficient estimation may not only make rescue aimless, but also delay the prime time. Rational estimation of disaster condition and corresponding material demand may ensure smooth rescue by providing material supports and avoiding the waste of relief resources. Estimation of relief goods demand after disaster is mainly divided into two aspects: estimation of disaster degree according to known condition, and estimation of material demand distribution according to disaster degree, economic condition, affected population distribution and other information.

The most present studies on relief goods demand after disaster aim at estimating single material. For example, on energy supply in disaster area, Jun Cao ${ }^{[1]}$, et al. gave energy supply strategy based on event-driven and feedback mechanism. In addition, attention is also paid to relevant estimation of special disaster. For example, on complicated analysis model of flood disaster, Huang Chongfu ${ }^{[2]}$, et al. put forward basic models related to climate factor and social factor. On deficient informationization of natural disaster monitoring technology, Sunhai ${ }^{[3]}$, et al. built an information system for urban comprehensive disaster assessment based on WebGIS. Qian Fenglin ${ }^{[4]}$, et al. imported BP neural network 
technique and improved prediction accuracy to predict affected population in earthquake. Fu Zhiyan ${ }^{[5]}$, et al. established case-based reasoning, key factor model with Euclidean distance to predict material demand after disaster. In conclusion, there are few researches on estimation of relief goods demand, without systematic resolution.

\section{Estimation of building damage level based on long-distance image}

Long-distance images, such as aerial photo or satellite images, are the first information obtained by rescuers after earthquake, tsunami and other large-scale disasters. By comparing these images, building damage in affected area can be easily and intuitively represented. Manual observation, affected by subjective factors, is always in low efficiency, so we need more objective evaluation in large affected area with complicated disaster condition. Therefore, it is necessary to study the comparison methods of long-distance images in different times to make objective evaluation of building damage in affected area.

After disaster, damaged buildings are different from original ones in shape, while undamaged buildings are different in color and texture with unchanged shape. Therefore, the basic idea of determining whether the building is damaged by its shape is formed. Major expression of shape in image is not only the edge information, but also the gradient information of image. Let long-distance images before and after disaster be $P$ and $P^{\prime}$, respectively, the difference between these two images $\mathrm{d}\left(P, P^{\prime}\right.$ can be expressed as follows:

Compared with the original image, edge information greatly simplifies the difficulty and complexity of the contrast. However, there is no definite matching relation between edges, so the calculation of edge difference is still a highdimensional calculation. In addition, the comparison result of simple edge similarity cannot show the damage level of building. For example, comparison between aerial photos and edge contrast in south beach of Haramachi, Minamisoma City, Fukushima before and after 9.0-magnitude earthquake on March 11 ${ }^{\text {th }}, 2011$ in Japan (hereinafter referred to 2011 Tohoku Earthquake) are as follows: 


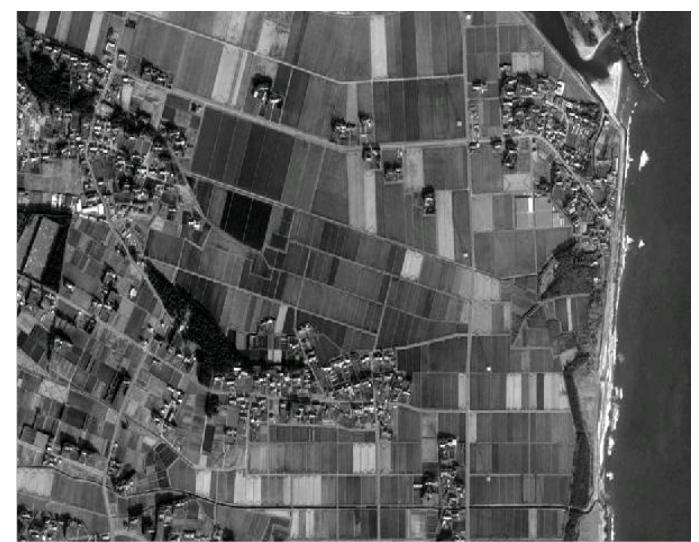

a) Image before earthquake

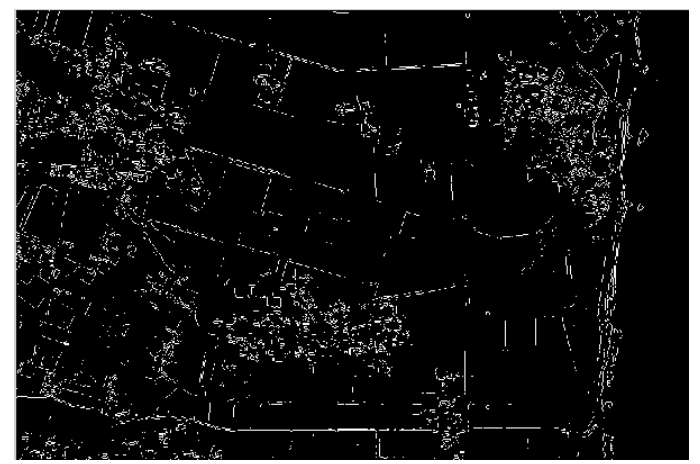

c) Image edge before earthquake

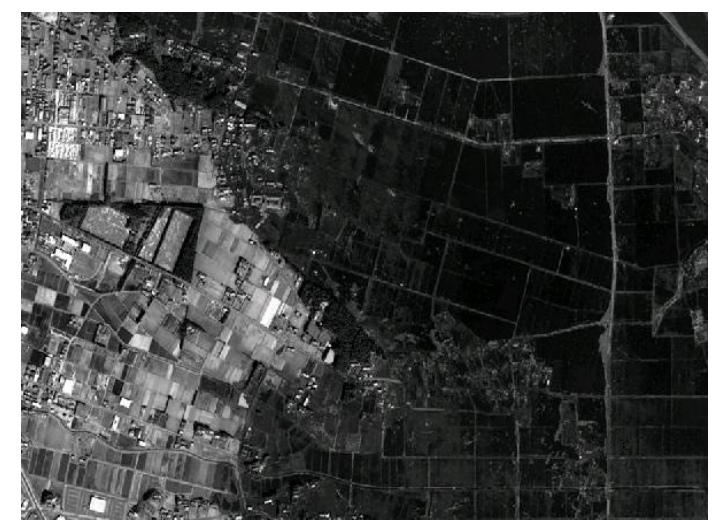

b) Image after earthquake

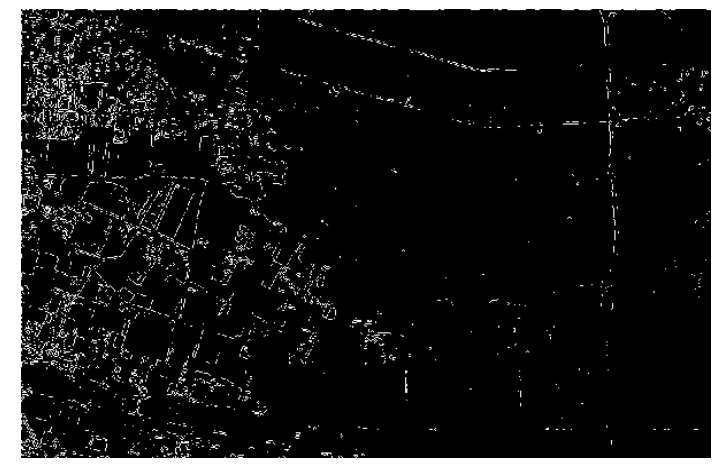

d) Image edge after earthquake

Fig. 1 Contrast of images and edges of south beach

With regular shape, such as square and circular arc, the appearance of modern building in aerial photo is regular quadrangle or circle, and the edge is segment or arc in principal. After being damaged, original quadrangle or circle will be broken, and the length and direction of remained segment or arc will be changed for the damaged architectural appearance. Let original length of one building edge be $\mathrm{L}$, the edge length between two ends after being damaged is always no less than L, which means: .

In this Formula, $\mathrm{E}$ is the original edge, while E' is the corresponding edge after disaster. In addition, new edges may occur in an area without edge. Therefore, it can be concluded that the more complicated the edge of damaged building is, the higher the damage level will be.

Edge complexity can be measured with two parameters of edge-direction value and location value. With these two values, we can obtain the number of line edge in image. The more sever the disaster is, the more complex the edge parameters are with shorter length, larger quantity and more different direction of the edges in long-distance image. Actually, all the edges in image can be expressed $y=k I x+b$

as:

The following formula can be obtained by transforming Equation 1-3 into polar 
form: $\rho=x \sin \theta+y \cos \theta$.

Coordinates $(x, y)$ in one same straight line correspond to the same parameter $(\rho, \theta)$, otherwise, coordinates will correspond to other parameters. That is, the distribution of corresponding parameters $(\rho, \theta)$ tends to be even when the lines in image are short and scattered, while the parameters are concentrated when coordinates are located in small amount of lines. With Hough transformation, the transformation from coordinate $(x, y)$ into parameter $(\rho, \theta)$ in image processing, line distribution in edge image can be obtained. Fig. 2 shows the contrast of aerial photos, edges and Hough transformations of Iigohama in Miyagi before and after 2011 Tohoku Earthquake:

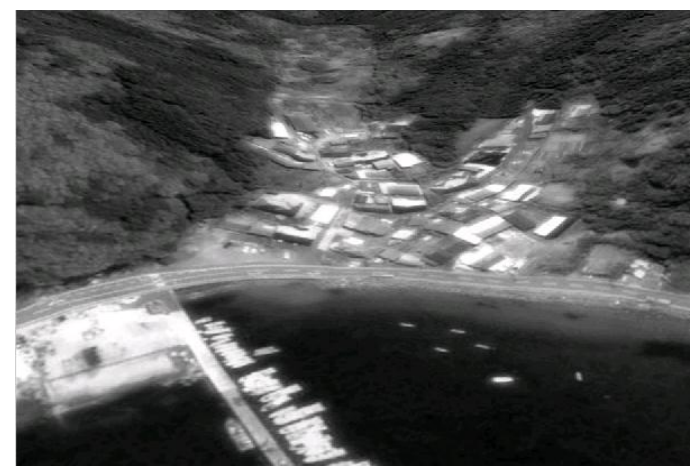

a. Image before earthquake

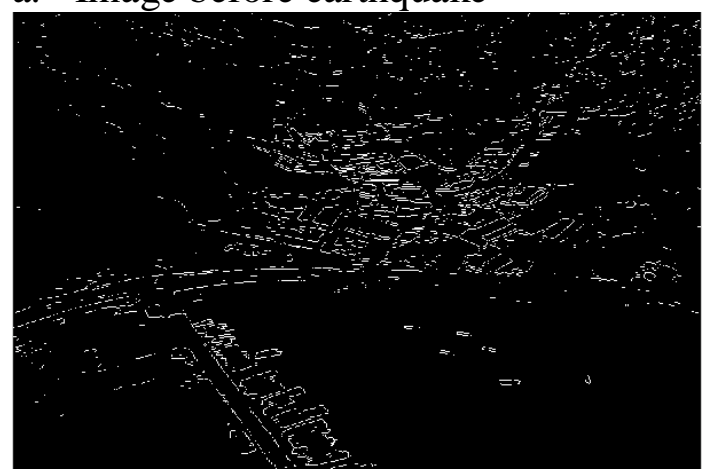

c. Edges in image before earthquake

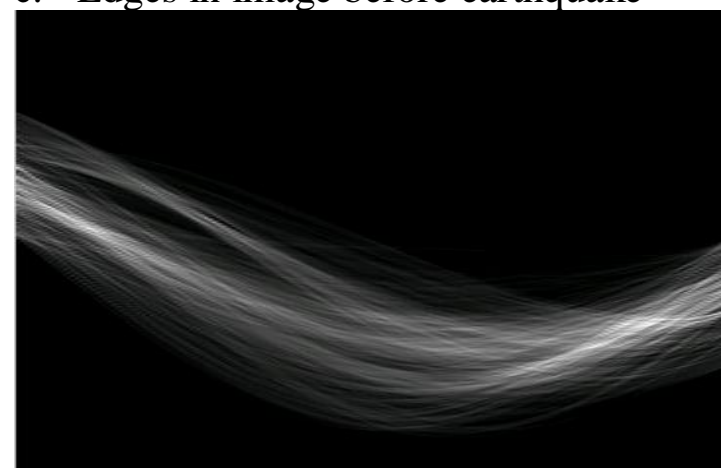

e. Hough transformation of image before earthquake

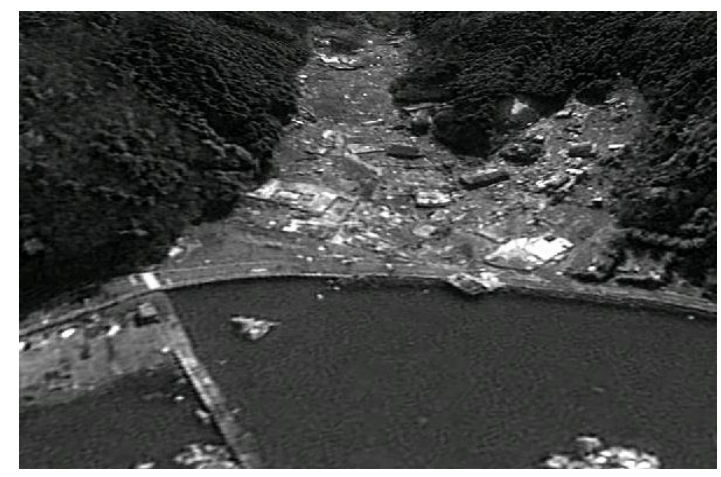

b. Image after earthquake

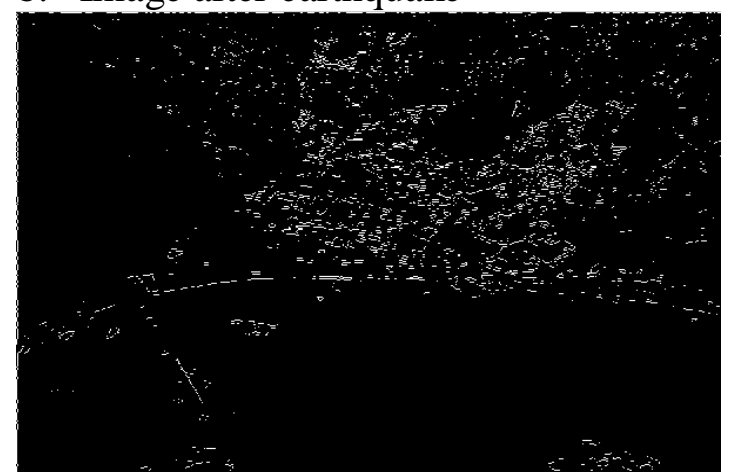

d. Edges in image after earthquake

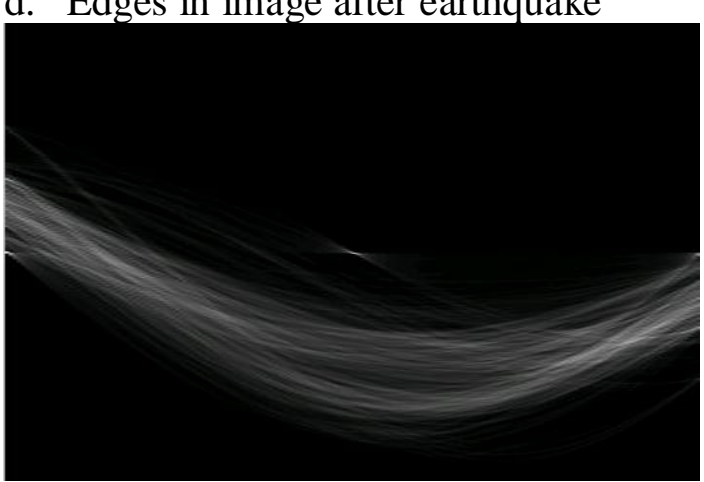

earthquake

Fig. 2 Contrast of Hough transformation for images of Iigohama in Miyagi before and after earthquake 
In Fig. 2, Sub-graph e and Sub-graph $\mathrm{f}$ show the Hough transformation for longdistance images of Iigohama in Miyagi before and after earthquake. Horizontal and vertical coordinates in the graph represent parameter $\rho$ and $\theta$, respectively, while the brightness represents the number of corresponding coordinate $(\mathrm{x}, \mathrm{y})$, which is the length of segment corresponding to the parameter. Fig. 2 shows that there is no obvious difference between two edge images to a great extent, but the parameters are much more concentrated in image before earthquake than after earthquake after Hough transformation.

Quantized standard is necessary for the objective evaluation of Hough parameter concentration ratio. The result of Hough transformation can be regarded as histogram in parameter plane, so the median line is introduced to measure the parameter concentration ratio. Counts, count $(\mathrm{P})$, in parameter plane (the pixel count in original image) should be counted and ranked from high to low. Then, parameter counts, count $(\rho, \theta, \alpha)$ accounting for a certain ratio $\alpha$ should be counted to obtain the ratio to all the non-zero parameter counts as measurement focus $(\alpha)$ :

$$
\left\{\begin{array}{l}
S=\left\{(\rho, \theta) \mid H(\rho, \theta)>H(\tilde{\rho}, \tilde{\theta}),(\tilde{\rho}, \tilde{\theta}) \in \bar{S}, \sum H(\rho, \theta)>\operatorname{count}(P) \alpha\right\} \\
f o c u s(\alpha)=\frac{\operatorname{count}((\rho, \theta) \mid(\rho, \theta) \in S)}{\operatorname{count}((\rho, \theta) \mid H(\rho, \theta)>0)}
\end{array}\right.
$$

In Formula, SI $\{(\rho, \theta)\}$, while $(\tilde{\rho}, \tilde{\theta})$ represents the parameter pairs making Hough transformation sum larger than $a$, that is, the parameter pairs except $\mathrm{S}$.

Edge concentration ratio is calculated for aerial photos, which are transformed in size 512 x 512 by bilinear interpolation, before and after 2011 Tohoku Earthquake according to above method. The results are shown in Table 1.

Table 1 Contrast of edge concentration ratio in images before and after 2011 Tohoku Earthquake

\begin{tabular}{llll}
\hline $\begin{array}{l}\text { Affected } \\
\text { area }\end{array}$ & $\alpha$ & $\begin{array}{l}\text { Before } \\
\text { earthquake } \\
\text { Focus }(\alpha)\end{array}$ & $\begin{array}{l}\text { After } \\
\text { earthquake } \\
\text { Focus }(\alpha)\end{array}$ \\
\hline $\begin{array}{l}\text { Ishinomaki } \\
\text { Natori }\end{array}$ & 0.01 & 0.0028 & 0.0022 \\
$\begin{array}{l}\text { Sendai } \\
\text { Airport } \\
\text { Arahama, }\end{array}$ & 0.02 & 0.0057 & 0.0056 \\
$\begin{array}{l}\text { Sendai } \\
\text { Fujitsuka, }\end{array}$ & 0.1 & 0.0441 & 0.0144 \\
$\begin{array}{l}\text { Sendai } \\
\text { Iwanuma- } \\
\text { Matsushima }\end{array}$ & 0.5 & 0.0942 & 0.0896 \\
\hline
\end{tabular}

Table 1 shows that the value of $\alpha$ is from 0.01 to 0.1 in calculation of concentration ratio for all areas, and the concentration ratio before earthquake is larger than that after earthquake. Therefore, it is feasible to measure damage level with concentration ratio.

\section{Estimation of affected population based on refugee information}

After disaster, detailed affected population is unclear because of population mobility. Therefore, it is difficult to estimate casualty and prepare relief goods. Correct estimation of affected population is necessary in precise demand estimation of relief goods after disaster. Let total population in affected area before disaster be $\mathrm{Hp}$, and probability density function 
for ratio of refugee $\mathrm{r}$ be $f(r)$, the refugees exposed to the edges of affected area after disaster $\mathrm{C}_{\mathrm{R}}$ can be expressed as follows:

Conversely, with the observed refugee population $C_{R}$, the estimation of total affected population is as follows:

$$
\begin{aligned}
\tilde{H}_{p} & =\arg \max _{C_{R}} P\left(C_{R} \mid H_{p}\right) \\
& =\arg \max _{C_{R}} \int_{0}^{\frac{C_{R}}{H_{p}}} f(r) d r
\end{aligned}
$$

By assuming that total population is subject to the distribution of density function $g(h)$, the maximum posterior estimation of $\mathrm{Hp}$ is:

$$
\begin{gathered}
\tilde{H}_{p, M A P}=\arg \max _{C_{R}} P\left(C_{R} \mid H_{p}\right) g\left(C_{R}\right) \\
=\arg \max _{C_{R}} \frac{P\left(C_{R} \mid H_{p}\right) g\left(C_{R}\right)}{\int_{R} P\left(C_{R} \mid r\right) g(r) d r}
\end{gathered}
$$

In Formula, $\mathbf{R}$ is the definitional domain of function $\mathrm{g}$.

Generally, distribution function of $\mathrm{Hp}$ can be fitted with existing information. For example, approximation can be realized by Gaussian distribution with permanent resident population as average value. Although psychological factors will fluctuate under the effects of disaster type and degree, it is still possible to obtain probability density function $f(r)$ with historic data statistics according to specific disaster type. The above formula can be used to estimate actual affected population in disaster area.

\section{Distribution estimation of relief goods demand}

According to the discussion in Part 2, total demand of relief goods can be estimated with refugee information, and demand distribution in area can be known with escape route. Escape process is also the process of demand information transmission when refugee population is the representative of demand information. Although there are few particular aimless exceptions, refugees will generally escape from affected area to the entrance. Taking residential zones as points and lines between residential zones, as well as residential zone and entrance as edges, the process of demand information transmission can be presented as a digraph shown in Fig. 3:

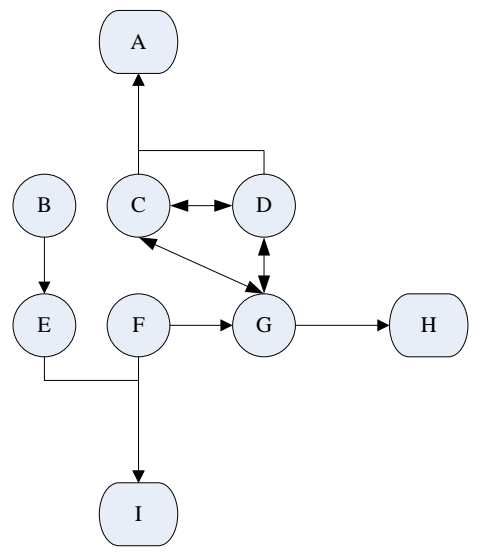

Fig. 3 Transmission map of relief goods demand

In Fig. 3, B, C, D, E, F and $\mathrm{G}$ are the exits of affected areas $\mathrm{A}, \mathrm{H}$ and $\mathrm{I}$, and the lines between these points represent escape direction, which is also the demand transmission direction. Demand information will be transmitted with overlap and interaction because each demand transmission exit may include information from multiple demand sources. These transmission exits, however, can still be distinguished.

The following assumption is made to eliminate mutual transmission (double sided 
arrow) during demand transmission: refugees will escape in direction close to the exit. Actually, there will be aimless escape at the beginning, but people can always get correct information about escape route after reverse population transmission. Consequently, a single route of information transmission from each node in image can be obtained by calculating the distance between every residential area and exit successively. Therefore, the assembled view can be transformed into a few trees with exits as root nodes and residential zones as leaf nodes. By simplifying, independent one-way trees can be separated from every exit as shown in Fig. 3.

In Fig. 3, residential zone is toward the only exit through directed edge, and simple trees are formed with points $\mathrm{A}, \mathrm{C}$ and $\mathrm{D}$, points $\mathrm{B}, \mathrm{E}$, $F$ and $I$, as well as points $G$ and $H$, respectively.

It seems that when refugee population in one city flows to the other, the estimated relief goods demand in the other city will grow with the increased refugees. In other words, material demand will accumulate in demand transmission tree. For example, the superficial demand in B is the sum of actual demands in A and B when the escape route between residential zones $\mathrm{A}$ and $\mathrm{B}$ is $\mathrm{A} \rightarrow \mathrm{B}$. In conclusion, the estimated demand at the exit in affected area is the sum in demand transmission tree. According to this characteristic, demand distribution algorithm with forward accumulation - backward calculation (BP demand distribution algorithm) is designed.

\section{Algorithm 2-2 (BP demand distribution algorithm)}

Step 1: Let demand transmission tree be $\mathrm{T}$, in which the leaf set is L. Mark the demand N(r) of all nodes $\mathrm{r}$ in $\mathrm{T}$ as fixed population $\mathrm{P}(\mathrm{r})$.

Step 2: Take any node $\mathrm{r} 0$ in $\mathrm{L}$, the neighboring node to which is r1. $\mathrm{N}(\mathrm{r} 1)=\mathrm{N}(\mathrm{r} 1)+\mathrm{N}(\mathrm{r} 0)$.

Step 3: Let $\mathrm{L}=\mathrm{L}-\{\mathrm{r} 0\}$. When there is no node toward $\mathrm{r} 1$ in $\mathrm{T}$ and $\mathrm{r} 1$ is not the root $\mathrm{rr}$, let $\mathrm{L}=\mathrm{LU}\{\mathrm{r} 1\}$. Turn to Step 4 if $\mathrm{L}$ is a null set, otherwise, turn to Step 2.

Step 4: Let $L=\{r r\}$, and suppose that $R(r r)$ is the estimation result for relief goods demand in node rr.

Step 5: Select any node $\mathrm{r} 0$ in $\mathrm{L}$ and all the sub-nodes $\mathrm{r} 1, \ldots$ rk of $\mathrm{r} 0$, and calculate demand estimation for all sub-nodes according to the coefficient $\mathrm{N}(\mathrm{r} 1), \ldots, \mathrm{N}(\mathrm{rk})$ (or estimate the maximum posterior probability with average value $\mathrm{N}(\mathrm{r} 1), \ldots, \mathrm{N}(\mathrm{rk})$ according to different distribution function):

Step 6: Let $\mathrm{L}=\mathrm{L}-\{\mathrm{r} 0\}$ and $\mathrm{L}=\mathrm{LU}\{\mathrm{r} 1, . . \mathrm{rk}\}$, and finish the estimation when $\mathrm{L}$ is null set, otherwise, turn to Step 5.

\section{CONCLUSIONS}

As a complex system engineering, the emergency rescue is based on demand estimation of relief goods. Total demand of relief goods was estimated by analyzing affected population according to damage level of buildings in long-distance images and refugee population. Material demand distribution was estimated with routes in affected area and 
refugee population at exits. This work put forward estimation method for building damage based on edge concentration ratio, estimation method for affected population based on posterior probability and demand distribution algorithm with backward calculation. The estimation of material demand after disaster was also systematically resolved. However, there are still some deficiencies during the research process, such as the lack of verification with actual disaster data for algorithm result, no practice of algorithm, etc. The work, therefore, will strive to contact with relevant units to obtain actual data for algorithm verification and establish an information system for more convenient use.

\section{REFERENCES}

[1] Quanhua Tang; Yan Zhou; Jine Lei; A median computing algorithm based on multi-level space compressed measureintegral[C]. IEEE Youth Conference on Information Computing and Telecommunications (YC-ICT), 2010: 97101.

[2] Chen Liwei, Tang Quanhua. Path selection algorithms for emergency logistics based time expectation[C]. ICLEM: Logistics for Sustained Economic Development, 2010(4): 3671-3678.

[3] Tao Wu, L. Shi, N.A. Duffie. An HNP-MP Approach for the Capacitated Multi-Item Lot Sizing Problem with Setup Times[J]. IEEE trans. Automation Science and Engineering, 2010, 7(3): 500-511. 